\title{
PREVALENCIA DE VIDA Y EDAD DE INICIO DE TRASTORNOS MENTALES EN EL PERÚ URBANO: RESULTADOS DEL ESTUDIO MUNDIAL DE SALUD MENTAL, 2005
}

\author{
Fabián Fiestas ${ }^{1, a}$, Marina Piazza $a^{1,2, b}$
}

\begin{abstract}
RESUMEN
Objetivos. Determinar la prevalencia de vida de 18 trastornos mentales y establecer el patrón que tienen dichos trastornos respecto a la edad de inicio, en cinco ciudades del Perú. Materiales y métodos. Como parte del Estudio Mundial de Salud Mental (EMSM), el estudio en Perú siguió un muestreo probabilístico, multietápico de personas entre 18 y 65 años de Lima Metropolitana, Chiclayo, Arequipa, Huancayo e Iquitos. Se administró la versión para computadora de la entrevista diagnóstica internacional compuesta (CIDI, Composite International Diagnostic Interview). Resultados. La prevalencia de vida de al menos un trastorno mental fue 29\% (Error Estándar, EE 1,2), y la prevalencia de al menos dos y tres trastornos fue $10,5 \%(E E 0,7)$ y $4 \%(E E 0,4)$, respectivamente. Los trastornos de ansiedad fueron más frecuentes, con una prevalencia de 14,9\% (EE 0,9), seguidos por los trastornos del humor con $8,2 \%$ (EE 0,5), trastornos de control de impulsos con 8,1\% (EE 0,8) y trastornos por consumo de sustancias $(5,8 \% ; E E, 0,3)$. La edad de inicio tuvo fue más temprana para los trastornos de ansiedad (15 años) y los trastornos del control de impulsos (20 años). Las cohortes más jóvenes tuvieron más riesgo de tener un trastorno mental. Conclusiones. Casi un tercio de la población urbana adulta de cinco ciudades del Perú ha tenido alguna enfermedad psiquiátrica a un momento dado en su vida, y la comorbilidad es frecuente. La mayoría de trastornos inician antes de los 30 años.
\end{abstract}

Palabras clave: Prevalencia; Epidemiología; Salud mental; Trastornos mentales (fuente: DeCS BIREME).

\section{LIFETIME PREVALENCE AND AGE OF ONSET OF MENTAL DISORDERS IN PERU: RESULTS OF THE WORLD MENTAL HEALTH STUDY, 2005}

\begin{abstract}
Objectives. To determine the lifetime prevalence of 18 mental disorders and to establish the pattern that those disorders have with the age of onset in five cities of Peru. Materials and methods. As part of the World Mental Health Survey, the study in Peru followed a probabilistic multistage sample of people between 18 and 65 years old in Lima, Chiclayo, Arequipa, Huancayo and Iquitos. The desktop version of the Composite International Diagnostic Interview (CIDI) was administered. Results. The lifetime prevalence of at least one mental disorder was 29\% (SE 1.2), and the prevalence of at least two or three was 10.5\% (SE 0.7) and 4\% (SE 0.4), respectively. Anxiety disorders were more common with $14.9 \%$ (SE 0.9) prevalence, followed by mood disorders with $8.2 \%$ (SE 0.5), impulse control disorders with $8.1 \%$ (SE 0.8), and substance use disorders (5.8\%; SE 0.3). The age of onset was earlier for anxiety disorders (15 years old) and for impulse control disorders (20 years old). Younger respondents were more likely to have a mental disorder. Conclusions. Almost a third of the adult population of five cities in Peru has had some psychiatric disorder at a given time in their lives, and comorbidity is common. Most disorders begin before age 30.
\end{abstract}

Key words: Prevalence; Epidemiology; Mental health; Mental disorders (source: MeSH NLM).

\footnotetext{
Unidad de Análisis y Generación de Evidencias en Salud Pública, Instituto Nacional de Salud. Lima, Perú. Lima, Perú

Facultad de Salud Pública y Administración, Universidad Peruana Cayetano Heredia. Lima, Perú

Médico epidemiólogo; ${ }^{\text {b }}$ Psicóloga, maestra en Salud Pública, doctora en Ciencias

Recibido: 12-06-13 Aprobado: 11-12-13
}

Citar como: Fiestas F, Piazza M. Prevalencia de vida y edad de inicio de transtornos mentales en el Perú urbano: Resultados del estudio mundial de salud mental, 2005. Rev Peru Med Exp Salud Publica. 2014;31(1):39-47. 


\section{INTRODUCCIÓN}

En el Perú se ha estimado que las enfermedades neuropsiquiátricas son responsables de aproximadamente 830000 años de vida perdidos por discapacidad o muerte prematura. Ello indica que este grupo de enfermedades son la primera causa de carga de enfermedad en el país ${ }^{(1)}$, con una carga similar a la que generan juntas las enfermedades cardiovasculares y el cáncer.

En el Perú existe evidencia de que los trastornos mentales son de alta prevalencia. Por ejemplo, estudios epidemiológicos hechos en varios ámbitos regionales peruanos entre el 2001 y 2005, han reportado prevalencias de vida para cualquier trastorno mental (definidos con el CIE-10) de 37,3\% para Lima Metropolitana ${ }^{(2)}$, y de $39,3 \%$ en ciudades de la sierra ${ }^{(3)}$ $y$ en ciudades de la selva ${ }^{(4)}$.

El presente estudio tiene como objetivo presentar los resultados de la Encuesta Mundial de Salud Mental para Perú (EMSM-Perú), realizada entre los años 2004 y 2005 , respecto a la prevalencia de vida de 18 trastornos mentales de cinco de las mayores ciudades del país. El hecho de que se usó la metodología estandarizada de la EMSM, hace que el presente estudio logre obtener resultados comparables con otros países de la región latinoamericana, y con otros países de bajos y medianos ingresos en el globo, lo cual ayuda a comprender mejor cómo se comportan los trastornos mentales en el Perú, desde una perspectiva regional y global.

\section{MATERIALES Y MÉTODOS}

Como parte de la Encuesta Mundial de Salud Mental, el Ministerio de Salud del Perú y el Instituto Nacional de Estadística e Informática implementaron la versión peruana de dicha encuesta en los años 2004 y 2005, siguiendo en general la metodología estándar y ampliamente descrita en los reportes suscitados de esta iniciativa mundial ${ }^{(6)}$.

\section{POBLACIÓN Y MUESTRA}

La población de estudio incluyó a residentes, adultos, de viviendas en la comunidad, que no corresponden a instituciones como hospitales, cuarteles, cárceles, entre otras. Las ciudades seleccionadas fueron Lima Metropolitana, la cual alberga un tercio de la población peruana, y otras cuatro ciudades como Chiclayo en la costa, Huancayo y Arequipa en la sierra, e Iquitos en la selva. En estas cinco ciudades viven la mitad de la población urbana del país, según datos del censo del $2007^{(7)}$.
Los participantes de la muestra fueron seleccionados siguiendo un diseño probabilístico trietápico. En la primera etapa se seleccionaron en cada una de las cinco ciudades las unidades primarias de muestreo (UPM), conformadas por conglomerados de aproximadamente 80 viviendas cada una. Usando la información del censo del año 1993 y la información del precenso del año 1999, el marco muestral en cada ciudad fue dividido en dos estratos. El primer subestrato incluyó conglomerados que ya existían en el censo de 1993, y el segundo subestrato incluyó conglomerados que aparecieron después del censo de 1993. La selección de las UPM fue hecha usando probabilidades proporcionales al tamaño de viviendas.

La segunda etapa del muestreo significó hacer una lista actualizada de todas las unidades de vivienda dentro de los límites del conglomerado seleccionado al momento de la implementación de la encuesta. Así, de esta lista se seleccionaron por muestreo aleatorio simple diez unidades de vivienda (unidad secundaria de muestreo) por cada conglomerado.

Finalmente, la unidad terciaria de muestreo, se refiere a un adulto viviendo en la casa, seleccionado de una lista de todos los adultos elegibles (esto es, hombres o mujeres, entre 18 y 65 años de edad, que hablen español) residentes de la vivienda seleccionada. Este fue elegido usando la tabla de Kish. No se permitió la sustitución de individuos que no pudieron ser ubicados. La muestra no incluyó las parejas conyugales de los participantes, ni se incluyeron participantes secundarios.

El tamaño muestral fue de 4560 personas (3376 en Lima, 256 en Chiclayo, 480 en Arequipa, 224 en Huancayo y 224 en lquitos). Este tamaño muestral no permite inferencias para cada ciudad por separado, sino para todas en conjunto. Para cumplir con estos requerimientos muestrales, se seleccionaron 422 UPM en Lima Metropolitana y 148 del resto de las cuatro ciudades. En general, para las cinco ciudades, la proporción de no respuesta fue de $9,8 \%$, siendo esta mayor en Lima Metropolitana $(11,8 \%)$ que en las otras cuatro ciudades $(3,9 \%)$.

El consentimiento informado y el protocolo del estudio fueron revisados y aprobados por el Comité de Ética del Instituto Nacional de Salud del Perú.

\section{EVALUACIONES E INSTRUMENTOS}

El estudio usó una versión para laptop del instrumento de la EMSM de la Organización Mundial de la Salud (OMS) llamado Composite International Diagnostic Interview (CIDI), traducido al español usando los procedimientos 
estándares de traducción recomendados por la OMS ${ }^{(8)}$, y utilizado en varios países latinoamericanos como México y Colombia, y otras poblaciones como los hispanos viviendo en los EE. UU., con buen desempeño ${ }^{(9-11)}$. Además de ello, en el Perú se realizó un proceso de armonización durante la prueba piloto donde se tomó en cuenta la redacción, contenido y estructura de las preguntas.

El cuestionario CIDI contiene 40 módulos con preguntas estandarizadas que agrupan a los trastornos psiquiátricos en cuatro categorías siguiendo los criterios del Manual Diagnóstico y Estadístico de Trastornos Mentales (DSM-IV) (12) : i) trastornos de ansiedad (trastorno del pánico, trastorno de ansiedad generalizada, fobia social, fobias específicas, pánico sin agorafobia, trastorno de ansiedad de separación, y trastorno de estrés postraumático); ii) trastornos del humor (trastorno depresivo mayor, distimia, trastorno bipolar I y II); iii) trastornos por uso de sustancias (abuso de alcohol, dependencia al alcohol, abuso de drogas y dependencia a drogas), y iv) trastornos del control de impulsos (trastorno oposicionista-desafiante, trastorno de conducta y trastorno de déficit de atención). Este último grupo de trastornos fue evaluado solo en aquellos entre 18 y 44 años de edad, por ser estos, trastornos de la niñez, adolescencia y adultez temprana, y por ello no afectarse la prevalencia de vida al poner esta restricción. Además, es importante señalar que el CIDI contiene una serie de reglas orgánicas de exclusión de módulos y reglas jerárquicas para aminorar la carga que significa la administración del instrumento en los participantes. En general, estas reglas tienen evidencia de ser altamente sensibles, con muy pocos casos positivos no detectados de trastornos mentales ${ }^{(13-16)}$.

Además, el instrumento CIDI contiene preguntas de edad de inicio de los trastornos psiquiátricos estandarizadas, con un diseño que evita que se obtengan respuestas no plausibles. En general, el patrón que siguen estas preguntas en el CIDI permite obtener la edad exacta en que el trastorno se presenta.

El CIDI usado para este estudio tuvo dos partes: La parte I, que contiene los trastornos de interés central, y fue aplicada a todos los participantes. Los diagnósticos incluidos en esta parte fueron los trastornos del humor (depresión mayor, distimia y trastorno bipolar), trastornos de ansiedad (trastorno del pánico, trastorno de ansiedad generalizada, fobia social, fobia específica y agorafobia sin pánico) y trastornos por uso de sustancias (abuso y dependencia a alcohol y drogas).

La parte II del instrumento incluye la valoración de diagnósticos como el trastorno de estrés postraumático, trastorno de ansiedad de separación, trastorno oposicionista-desafiante, trastorno de conducta, trastorno de hiperactividad y déficit de atención y el trastorno explosivo intermitente. Esta parte del CIDI fue aplicada a una submuestra probabilística de los participantes de la parte I, los cuales fueron seleccionados usando un algoritmo computarizado. Específicamente, las reglas del algoritmo permitieron elegir para la parte II a aquellos que cumplían criterios para cualquiera de los trastornos psiquiátricos de la parte I y también a una submuestra de todos los demás (25\%).

Los instrumentos fueron aplicados por 40 encuestadores capacitados, egresados o bachilleres universitarios. Las actividades de capacitación se llevaron a cabo en 11 días, en las que en un total de 88 horas recibieron lecciones del marco conceptual del estudio, sesiones clínicas prácticas, práctica de campo y evaluación final.

\section{ANÁLISIS ESTADÍSTICO}

El análisis de los datos tomó en cuenta la complejidad del diseño del muestreo, usando ponderaciones para ajustar por las probabilidades diferenciadas de selección que resultaron de la metodología de muestreo estratificado multietápico de los participantes, así como también ajustar por la proporción de no respuesta. Se realizó, además, una ponderación posestratificación para ajustar la muestra a las características de la población en las edades correspondientes y sexo, usando el precenso de 1999 de las ciudades participantes.

Así, debido a que todos los participantes completaron la parte I, los datos de esta parte se ponderaron para ajustar las probabilidades diferenciadas de selección entre y dentro de viviendas y para consensuar las distribuciones de la muestra con las distribuciones de la población respecto a variables demográficas clave y a los datos geográficos. Por su parte, los datos de la parte II fueron ponderados de acuerdo a los criterios de selección descritos anteriormente. Como resultado de la complejidad del muestreo y probabilidades de selección, los errores estándar de todos los estimados estadísticos para este reporte fueron obtenidos usando el método de linearización de Taylor con el paquete estadístico Sudaan release 8.0.1 for Windows (http://www.rti.org/sudaan).

Para la estimación de la prevalencia de vida se uso la proporción de personas que hayan tenido un trastorno dado en cualquier momento a la fecha de la entrevista (incidencia acumulada). La edad de inicio y el riesgo proyectado de tener alguna vez en la vida un trastorno mental al llegar a los 65 años fueron estimados usando el método actuarial de dos partes implementado en SAS 8.2 para Windows. La valoración de posibles predictores sociodemográficos y el efecto de cohorte fue realizada mediante el método de análisis de sobreviva de tiempos 
discretos, usando como unidad de análisis el añopersona ${ }^{(17)}$. El error de tipo I (alfa) fue establecido en 0,05 a dos colas. Los errores estándar para los estimados de riesgos de vida fueron calculados usando el método de jacknife de repetidas replicaciones implementado con un macro en SAS ${ }^{(18)}$.

\section{RESULTADOS}

\section{PREVALENCIA DE VIDA}

La muestra incluyó 3930 sujetos, de los cuales 51,6\% fueron mujeres, y aproximadamente $50 \%$ estuvieron entre 18 y 34 años. Como se visualiza en la Tabla 1, al momento de la entrevista, $29 \%$ de los participantes tuvieron al menos un trastorno psiquiátrico en su vida;
$10,5 \%$ tuvieron dos o más diagnósticos y $4 \%$, tres o más trastornos mentales. Los trastornos de ansiedad fueron los más comunes, con $14,9 \%$ de prevalencia, seguidos de los trastornos del humor con $8,2 \%$ y trastornos por control de impulsos con $8,1 \%$, mientras que los trastornos por consumo de sustancias tuvieron una prevalencia de $5,8 \%$. Los cuatro trastornos individuales más comunes fueron las fobias específicas $(6,6 \%)$, depresión mayor $(6,4 \%)$, los trastornos de ansiedad de separación $(6,1 \%)$ y los trastornos por uso de alcohol (5,6\%).

La distribución por edad de estos trastornos resulta, por lo general, homogénea. Sin embargo, se observan diferencias para el trastorno del pánico, siendo las personas entre 45 a 54 años las que tuvieron menor prevalencia de estos trastornos respecto a los otros grupos de edad. El grupo de 18 a 29 años de edad

Tabla 1. Prevalencia de vida de trastornos psiquiátricos DSM-IV en el Perú

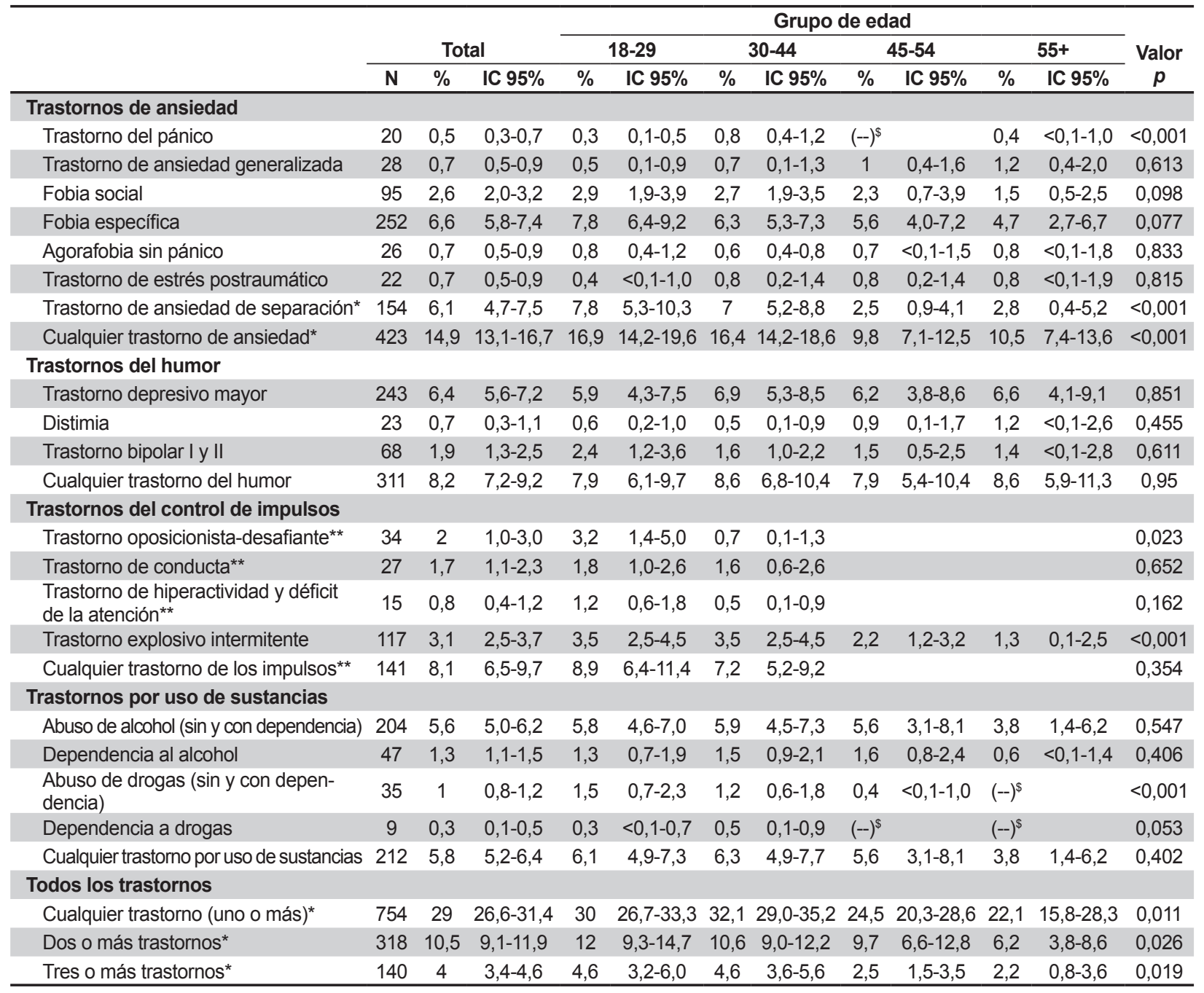

EE: error estándar. * Trastorno evaluado en la Parte $2,{ }^{* *}$ Trastorno evaluado en la Parte 2 en personas de 44 años o menos, $\$$ Valores no estimados debido a que el tamaño de muestra no lo permitió, Tamaño de muestra de Parte $1=3930$, Tamaño de muestra de Parte $1=1801$, Tamaño de muestra de Parte 2 y $<=44$ años de edad $=1287$ 
Tabla 2. Percentiles de la edad de inicio de trastornos psiquiátricos DSM-IV y riesgo de vida proyectado a la edad de 65 años

\begin{tabular}{|c|c|c|c|c|c|c|c|c|c|c|}
\hline \multirow[t]{2}{*}{ Trastorno } & \multicolumn{8}{|c|}{ Percentiles de la edad de inicio } & \multicolumn{2}{|c|}{$\begin{array}{c}\text { Riesgo de vida } \\
\text { proyectado a la edad de } \\
65 \text { años }\end{array}$} \\
\hline & 5 & 10 & 25 & 50 & 75 & 90 & 95 & 99 & $\%$ & EE \\
\hline \multicolumn{11}{|l|}{ Trastornos de ansiedad } \\
\hline Fobia social & 9 & 10 & 13 & 16 & 19 & 27 & 34 & 41 & 2,7 & $2,1-3,3$ \\
\hline Fobia específica & 5 & 5 & 7 & 10 & 13 & 20 & 27 & 36 & 6,8 & $6,0-7,6$ \\
\hline Trastorno de ansiedad de separación* & 6 & 8 & 14 & 24 & 37 & 57 & 57 & 57 & 8,5 & $6,0-11,0$ \\
\hline Cualquier trastorno de ansiedad* & 5 & 6 & 9 & 15 & 27 & 43 & 57 & 59 & 17,8 & $14,9-20,7$ \\
\hline \multicolumn{11}{|l|}{ Trastornos del humor } \\
\hline Trastorno depresivo mayor & 14 & 19 & 24 & 38 & 51 & 59 & 59 & 61 & 12,3 & $10,5-14,0$ \\
\hline Trastorno bipolar I y II & 15 & 17 & 19 & 25 & 37 & 41 & 46 & 51 & 2,6 & $2,0-3,2$ \\
\hline Cualquier trastorno del humor** & 14 & 18 & 22 & 35 & 47 & 58 & 59 & 61 & 14,7 & $12,7-16,7$ \\
\hline \multicolumn{11}{|l|}{ Trastornos del control de impulsos } \\
\hline Trastorno oposicionista-desafiante ${ }^{* *}$ & 7 & 7 & 8 & 13 & 15 & 17 & 17 & 18 & 2 & $1,0-3,0$ \\
\hline Trastorno explosivo intermitente & 9 & 12 & 17 & 26 & 37 & 61 & 61 & 61 & 4,4 & $2,8-6,0$ \\
\hline Cualquier trastorno de los impulsos ${ }^{* *}$ & 7 & 8 & 13 & 20 & 37 & 61 & 61 & 61 & 9,2 & $5,9-12,5$ \\
\hline \multicolumn{11}{|l|}{ Trastornos por uso de sustancias } \\
\hline Abuso de alcohol (sin y con dependencia) & 17 & 19 & 21 & 26 & 35 & 44 & 46 & 49 & 7,8 & $6,8-8,9$ \\
\hline Dependencia al alcohol & 16 & 18 & 21 & 26 & 36 & 39 & 40 & 45 & 1,8 & $1,4-2,2$ \\
\hline Abuso de drogas (sin y con dependencia) & 13 & 16 & 17 & 20 & 24 & 28 & 28 & 31 & 1,1 & $0,7-1,5$ \\
\hline Cualquier trastorno por uso de sustancias & 16 & 18 & 20 & 26 & 34 & 44 & 46 & 49 & 8 & $7,0-9,0$ \\
\hline \multicolumn{11}{|l|}{ Todos los trastornos } \\
\hline Cualquier trastorno (uno o más)* & 6 & 8 & 13 & 22 & 37 & 54 & 59 & 61 & 39,2 & $34,9-43,5$ \\
\hline
\end{tabular}

* Trastorno evaluado en la Parte 2, ** Trastorno evaluado en la Parte 2 en personas de 44 años o menos, Tamaño de muestra de Parte 1 = 3930 , Tamaño de muestra de Parte $1=1801$, Tamaño de muestra de Parte 2 y $<=44$ años de edad $=1287$

presenta mayor prevalencia que otros grupos etarios de trastornos de ansiedad de separación, de ira explosiva y de trastornos por consumo de drogas.

\section{EDAD DE INICIO Y RIESGO DE VIDA}

Como se muestra en la Tabla 2, la mediana (percentil 50) de la edad de inicio para cualquiera de los trastornos fue de 22 años, siendo los trastornos de ansiedad los que se presentan una tendencia de presentarse a una edad más temprana (mediana, 15 años), seguido por los trastornos de control de impulsos (20 años) y de los trastornos por uso de sustancias (26 años). En cambio, los trastornos del humor, aparecen a una edad más tardía, con una mediada a los 35 años. Dentro de este grupo de trastornos, la depresión mayor es la de inicia a una edad más tardía, con una mediana de 38 años.

Respecto al riesgo proyectado de haber tenido cualquiera de los trastornos en algún momento de su vida al alcanzar la edad de 65 años, se estimó que $39,2 \%$ de la población habrá padecido de alguna de estas enfermedades. Más específicamente, como también se ve en la Tabla 2 , el $17,8 \%$ de la población habrá tenido a algún trastorno de ansiedad antes de los 65 años, 14,7\% trastornos del humor, 9,2\% trastornos del control de impulsos, y $8 \%$ trastornos por uso de sustancias.

\section{EFECTO DE COHORTE}

En general, la Tabla 3 muestra que la generación más joven (de 18 a 29 años) está en mayor riesgo de padecer todos los trastornos mentales, individualmente o agrupados, en comparación con la generación de 55 años o más. Se evidencia, además, una tendencia de asociación tipo dosis-respuesta, en la que el riesgo de padecer un trastorno mental disminuye conforme es mayor la edad. Por ejemplo, se muestra que el odds que una persona pertenezca a la cohorte de 18-29 años tenga cualquier trastorno mental es 3,9 el odds que una persona de la cohorte de 55 a más años. Luego, para alguien de la cohorte de 30-44 años, o de la cohorte de 45-54 años, el odds es 2,7 y 1,4 veces, respectivamente, el odds de los de la cohorte de 55 a más años de tener cualquiera de los trastornos estudiados.

\section{FACTORES PREDICTORES DE LOS TRASTORNOS}

En la Tabla 4 se muestran los resultados de sobrevida de tiempos discretos que evalúan la asociación entre el nivel de educación, el sexo la edad y el riesgo de vida de 
Tabla 3. Edad al momento de la entrevista como predictor del riesgo de vida de trastornos psiquiátricos DSM-IV*

\begin{tabular}{|c|c|c|c|c|c|c|c|}
\hline \multirow{3}{*}{ Trastornos } & \multicolumn{6}{|c|}{ Grupos de edad comparados con aquellos de 55 años o más } & \multirow[b]{3}{*}{$\begin{array}{c}\text { Valor } \\
p\end{array}$} \\
\hline & \multicolumn{2}{|c|}{$18-29$} & \multicolumn{2}{|c|}{$30-44$} & \multicolumn{2}{|c|}{$45-54$} & \\
\hline & OR & IC 95\% & OR & IC $95 \%$ & OR & IC $95 \%$ & \\
\hline \multicolumn{8}{|l|}{ Trastornos de ansiedad } \\
\hline Fobia social & $2,4^{*}$ & $1,2-4,6$ & 1,9 & $1,0-3,7$ & 1,6 & $0,6-4,3$ & 0,033 \\
\hline Fobia específica & $1,8^{*}$ & $1,1-3,0$ & 1,4 & $0,9-2,0$ & 1,2 & $0,7-2,1$ & 0,022 \\
\hline Trastorno de ansiedad de separación ${ }^{\star *}$ & $10,6^{*}$ & $5,0-22,4$ & $5,5^{*}$ & $2,6-11,8$ & 1,2 & $0,4-3,2$ & $<0,001$ \\
\hline Cualquier trastorno de ansiedad ${ }^{* *}$ & $2,7^{*}$ & $1,7-4,1$ & $2,1^{*}$ & $1,4-3,2$ & 1 & $0,7-1,6$ & $<0,001$ \\
\hline \multicolumn{8}{|l|}{ Trastornos del humor } \\
\hline Trastorno depresivo mayor & $6,8^{*}$ & $3,9-11,9$ & $3,2^{*}$ & $1,6-6,4$ & 1,4 & $0,7-2,8$ & $<0,001$ \\
\hline Trastorno bipolar I y II & $6,1^{*}$ & $1,1-34,1$ & 1,7 & $0,5-6,3$ & 1,1 & $0,2-5,0$ & $<0,001$ \\
\hline Cualquier trastorno depresivo & $5,5^{*}$ & $3,1-9,8$ & $2,5^{*}$ & $1,3-4,7$ & 1,3 & $0,7-2,3$ & $<0,001$ \\
\hline \multicolumn{8}{|l|}{ Trastornos del control de impulsos } \\
\hline Trastorno oposicionista-desafiante*** & $4,3^{*}$ & $1,5-12,5$ & 1 & -- & & -- & 0,004 \\
\hline Trastorno explosivo intermitente & $8,4^{*}$ & $3,3-21,3$ & $5,0^{*}$ & $1,7-14,3$ & 2,4 & $0,8-7,0$ & $<0,001$ \\
\hline Cualquier trastorno de los impulsos ${ }^{\star * *}$ & $1,7^{*}$ & $1,1-2,7$ & 1 & -- & -- & -- & 0,01 \\
\hline \multicolumn{8}{|l|}{ Trastornos por uso de sustancias } \\
\hline Abuso de alcohol (sin y con dependencia) & $4,9^{*}$ & $2,6-9,5$ & 2,2 & $1,0-5,1$ & 1,5 & $0,6-3,7$ & $<0,001$ \\
\hline Dependencia al alcohol & $7,1^{*}$ & $1,6-31,4$ & 3,3 & $0,7-16,5$ & 2,8 & $0,5-14,9$ & $<0,001$ \\
\hline Abuso de drogas (sin y con dependencia) & $5,0^{*}$ & $1,0-24,4$ & 3 & $0,8-11,8$ & 1 & -- & 0,098 \\
\hline Cualquier trastorno por uso de sustancias & $4,8^{*}$ & $2,5-9,2$ & $2,3^{*}$ & $1,0-5,2$ & 1,5 & $0,6-3,7$ & $<0,001$ \\
\hline \multicolumn{8}{|l|}{ Todos los trastornos } \\
\hline Cualquier trastorno (uno o más) ${ }^{\star *}$ & $3,9^{*}$ & $2,6-5,8$ & $2,7^{*}$ & $1,8-3,9$ & 1,4 & $1,0-2,0$ & $<0,001$ \\
\hline
\end{tabular}

$¥$ Basado en modelos de sobrevivencia de tiempos discretos con la persona-año como la unidad de análisis

* valor $p<0,05,{ }^{* *}$ Trastorno evaluado en la Parte $2,{ }^{* * *}$ Trastorno evaluado en la Parte 2 en personas de 44 años o menos

los trastornos estudiados. Comparando la distribución de los trastornos por nivel educativo, se observa que el ser estudiante estuvo asociado a un menor riesgo de ansiedad que aquellos que solo alcanzaron primaria completa y que aquellos que completaron la secundaria, o mayores niveles educativos. Por su parte, el tener un nivel educativo menor a secundaria estuvo asociado a menor riesgo de trastornos por uso de sustancias. En relación al sexo, las mujeres tuvieron mayor probabilidad de presentar trastornos ansiosos, pero menor probabilidad de trastornos por uso de sustancias. Respecto a la edad, las generaciones más jóvenes (18-29 y 29-34 años) estuvieron asociadas a un mayor riesgo de vida de tener todos los grupos de trastornos, independientemente del sexo y el nivel educativo.

Tabla 4. Factores sociodemográficos y su asociación con los trastornos psiquiátricos DSM-IV*

\begin{tabular}{|c|c|c|c|c|c|c|c|c|}
\hline & \multicolumn{2}{|c|}{ Ansiedad } & \multicolumn{2}{|c|}{ Trastornos del humor } & \multicolumn{2}{|c|}{$\begin{array}{l}\text { Trastornos del control } \\
\text { de impulsos }\end{array}$} & \multicolumn{2}{|c|}{$\begin{array}{l}\text { Trastornos por uso } \\
\text { de sustancias }\end{array}$} \\
\hline & OR & IC $95 \%$ & OR & IC $95 \%$ & OR & IC $95 \%$ & OR & IC $95 \%$ \\
\hline \multicolumn{9}{|l|}{ Nivel de educación ${ }^{* *}$} \\
\hline Estudiante $^{* * *}$ & $0,6^{*}$ & $0,3-0,9$ & 0,7 & $0,4-1,4$ & 1,6 & $0,7-3,7$ & 0,8 & $0,4-1,5$ \\
\hline Menos de secundaria & $0,6^{*}$ & $0,5-0,9$ & 0,9 & $0,6-1,4$ & 1,3 & $0,5-3,4$ & $1,8^{*}$ & $1,1-3,1$ \\
\hline Secundaria completa o más & 1 & & 1 & & 1 & & 1 & \\
\hline \multicolumn{9}{|l|}{ Sexo } \\
\hline Mujer & $1,4^{*}$ & $1,1-1,7$ & 1 & $0,7-1,3$ & 0,8 & $0,5-1,2$ & $0,1^{*}$ & $<0,1-0,1$ \\
\hline Hombre & 1 & & 1 & & 1 & & 1 & \\
\hline \multicolumn{9}{|l|}{ Edad } \\
\hline $18-29$ & $2,5^{*}$ & $1,5-4,2$ & $4,8^{*}$ & $2,2-10,7$ & $32,4^{*}$ & $6,9-153,3$ & $9,4^{*}$ & $3,1-28,4$ \\
\hline $30-44$ & $2,0^{*}$ & $1,2-3,3$ & $2,2^{*}$ & $1,0-4,8$ & $18,8^{*}$ & $4,1-87,5$ & $5,0^{*}$ & $1,6-15,2$ \\
\hline $45-54$ & 1,1 & $0,6-2,0$ & 1,1 & $0,3-3,5$ & $8,2^{*}$ & $1,5-44,2$ & 2,3 & $0,7-7,3$ \\
\hline$\geq 55$ & 1 & & 1 & & 1 & & 1 & \\
\hline
\end{tabular}

* Basado en modelos de sobrevivencia de tiempos discretos con la persona-año como la unidad de análisis

* valor $\mathrm{P}<0,05,{ }^{* *}$ Predictor variable en el tiempo, ${ }^{* * *}$ Persona aún estudiando al momento de la entrevista 


\section{DISCUSIÓN}

La encuesta realizada en cinco de las ciudades más populosas de la costa, sierra y selva del Perú, encuentra que aproximadamente uno de cada tres personas ha tenido un trastorno mental a un momento dado, y que uno de cada diez ha tenido dos o más de estos trastornos. Los trastornos de ansiedad son los más comunes, seguidos por los trastornos del humor y del control de impulsos, aunque los trastornos por uso de sustancias mantienen una prevalencia importante en las poblaciones estudiadas. En general, se encontró también que los trastornos inician dentro de los 25 o 26 años de vida, a excepción de la depresión mayor, que tienen un patrón de iniciación más tardía, lo que a su vez causa que el riesgo de vida de tener un trastorno del humor en algún momento antes de los 65 años sea mayor que la prevalencia a un momento dado. Además, en este estudio se encuentra que las generaciones más jóvenes se encuentran en mayor riesgo de padecer cualquiera de los trastornos estudiados y que el sexo y el nivel de educación se asocian con patrones específicos a determinados trastornos.

Algunos aspectos deben ser tomados en cuenta para interpretar los resultados de la presente investigación. Por ejemplo, si bien se ha generado información de prevalencias para cinco de las ciudades más grandes del país, va a ser importante que futuros estudios con similar rigurosidad de diseño se lleven a cabo con una muestra de representatividad nacional, tanto para el área urbana como para el área rural. Respecto al instrumento CIDI, este ha sido rigurosamente validado y calibrado en diferentes contextos e idiomas, incluido el español ${ }^{(6,19,20)}$. Así, la versión en español del instrumento ha sido utilizada con buen funcionamiento en México ${ }^{(9)}$, Colombia ${ }^{(21)}$ y en la comunidad latina en los EE.UU. (11,22). Por otro lado, existen dos aspectos que pudieran estar traduciéndose en un subreporte de las prevalencias como los errores de medición relacionados con la memoria a los que encuestas epidemiológicas de autorreporte están predispuestas, y el hecho de que solo se incluyan personas no institucionalizadas en lugares como hospitales, cárceles, albergues, entre otras instituciones, donde los problemas de salud mental son frecuentes ${ }^{(6,10)}$.

Por otra parte, el estudio presenta varias fortalezas que ayudan a la validez de sus resultados. Primero, este es parte de la encuesta mundial de salud mental, en la que han participado hasta ahora más de veinte países, y en donde se han seguido en general diseños epidemiológicos estandarizados y métodos de implementación similares lo que permite una comparación con otros contextos nacionales, que a su vez permite tener puntos de referencia independientes que ayudan a interpretar los resultados obtenidos. Segundo, se utiliza una metodología probabilística de tres etapas, que termina con la selección de un único individuo por vivienda seleccionada, lo que es ampliamente aceptado como óptimo para establecer prevalencias poblacionales ${ }^{(6)}$. $Y$, tercero, se utiliza un instrumento estandarizado y probado en campo en poblaciones similares a la peruana, como son México y Colombia, y se logró un excelente nivel de participación.

Este es uno de los primeros reportes de resultados de la encuesta mundial de salud mental para el Perú. Cuando se compara las prevalencias obtenidas con la de otros países de la región como México ${ }^{(9)}$ y Colombia ${ }^{(21)}$ se hace evidente que existe una general similitud de las prevalencias de vida para la mayoría de trastornos, definidos con el DSM-IV, especialmente con México. Sin embargo, es muy notoria la diferencia entre Perú y Colombia respecto a la prevalencia de trastornos por uso de sustancias, que en Colombia es casi tres veces la prevalencia estimada para el Perú. Un análisis más detallado de las características y razones de estas diferencias se están realizando actualmente por nuestro grupo de investigación. Asimismo, como ha sido también notado en los reportes de los otros países latinoamericanos, las prevalencias y los patrones de prevalencias para trastornos DSM-IV individuales, varían notoriamente con los encontrados en países de otras latitudes, incluidos EE.UU., y los países europeos, asiáticos, africanos y oceánicos ${ }^{(5,23)}$. Las razones de estas diferencias no han sido exploradas en el presente estudio. Sin embargo, diferencias culturales que influyen tanto en la presentación de los trastornos como en su medición han sido propuestas y permanecen por ser estudiadas a mayor profundidad ${ }^{(9,24)}$

Respecto a la edad de inicio, los efectos de cohorte y los factores asociados, estos siguen también en general un patrón similar al encontrado en los otros países participantes de la encuesta mundial, aunque serán necesarios estudios ad hoc para entender las razones de las diferencias o similitudes con otros países.

Comparando los resultados con las prevalencias de vida reportadas por los estudios epidemiológicos realizados por el Instituto Nacional de Salud Mental peruano (INSM) durante casi el mismo quinquenio, (2-4) se encuentran diferencias. Por ejemplo, los resultados EMSP-Perú muestran menores prevalencias de cualquier trastorno $(29 \%)$, mientras que los estudios epidemiológicos en Lima Metropolitana, tres ciudades de la sierra, y en tres ciudades de la selva, encontraron prevalencias de vida para cualquier trastorno entre 37 y $39 \%{ }^{(2-4)}$. Similarmente, en el caso de depresión, mientras que la EMSM-Perú estima la prevalencia 
de vida en $6,4 \%$, los estudios epidemiológicos mencionados muestran prevalencias de vida entre 16 y $21 \%{ }^{(2-4)}$. Podemos mencionar entre las razones para explicar estas diferencias a los diferentes sistemas de diagnóstico utilizados en los estudios epidemiológicos mencionados y la presente encuesta. Específicamente, la EMSM-Perú usa el CIDI y las prevalencias reportadas aquí siguen el sistema DSM-IV, mientras que los otros estudios epidemiológicos utilizaron el instrumento MINI (Mini International Neuropsychiatric Interview) que usa el sistema diagnóstico CIE-10.

Otras diferencias metodológicas entre la EMSM-Perú y los estudios epidemiológicos del INSM, incluyen el método para seleccionar la muestra y las características de la población objetivo. Así, mientras que la EMSMPerú usa un diseño trietápico, con conglomerados y viviendas seleccionadas aleatoriamente y con un solo individuo por vivienda, los estudios del INSM usan un diseño bietápico, con selección de viviendas consecutivas y de varios participantes por vivienda, lo que también pueden ayudar a explicar los diferentes resultados. Además, las regiones incluidas en ambos estudios pueden diferir en términos de niveles de pobreza y exposición a factores estructurales como es la violencia política que azotó regiones peruanas, especialmente de la sierra central, durante la década de los 80. No obstante estas diferencias encontradas con los estudios epidemiológicos del INSM, los resultados de la EMSM-Perú coinciden en gran parte con las prevalencias y patrones hallados en los estudios en otros países de la región, que utilizan la misma metodología epidemiológica, esto brinda confianza acerca de la validez y comparabilidad de nuestros resultados con lo que se va conociendo a nivel global de los trastornos mentales.

Casi un tercio de la población urbana en el Perú sufre de alguna enfermedad psiquiátrica, y casi uno de cada diez personas presenta dos o más trastornos mentales a lo largo de su vida. La distribución de las prevalencias y las edades de inicio se condicen en general con lo encontrado en países en el mundo donde se implementó la Encuesta Mundial de Salud Mental, aunque futuros estudios deberán esclarecer diferencias incluso entre países de una misma región.

\begin{abstract}
Agradecimientos: al personal de los Centros de Coordinación de Recojo y Análisis de datos del WMH el apoyo en la implementación del trabajo de campo y consultoría en el análisis de datos. Agradecemos a Ron Kessler, Nancy Sampson y Colleen Bouzan (Universidad de Harvard), María Elena Medina Mora y Guilherme Borges (Instituto Mexicano de Psiquiatría), Jorge Rodríguez y María Edith Baca (Organización Panamericana de la Salud), Sergio Aguilar Gaxiola (Universidad Estatal de California en Fresno) y al equipo técnico local (conformado por funcionarios del MINSA, del Instituto Nacional de Salud Mental Honorio Delgado-Hideyo Noguchi y del INEI), el cual participó en la revisión del instrumento durante la fase inicial del estudio.
\end{abstract}

Contribuciones de autoría: FF y MP participaron de la ideación de estudio. FF escribió el primer borrador del artículo, con contribución significativa de MP. Ambos autores toman responsabilidad de la publicación.

Fuentes de financiamiento: la Encuesta Mundial de Salud Mental en Perú se desarrolla en colaboración con la Encuesta Mundial de Salud Mental (WMH) de la Organización Mundial de la Salud, la cual contó con financiamiento del Instituto Nacional de Salud Mental de los Estados Unidos (NIMH; R01 MH070884), la Fundación John D. y Catherine T. MacArthur, la Fundación Pfizer, el Servicio de Salud Pública de Estados Unidos (R13-MH066849, R01-MH069864, y R01 DA016558), el Centro Internacional Fogarty (FIRCA R03-TW006481), la Organización Panamericana de la Salud, y las compañías farmacéuticas Eli Lilly, Ortho-McNeil, GlaxoSmithKline, y BristolMyers Squibb. La Encuesta Mundial de Salud Mental en Perú es financiado por el Instituto Nacional de Salud del Ministerio de Salud del Perú, con apoyo adicional de la Organización Panamericana de la Salud.

Conflicto de interés: los autores declaran no tener conflictos de interés.

\section{REFERENCIAS BIBLIOGRÁFICAS}

1. Velásquez A. La carga de enfermedad y lesiones en el Perú y las otras prioridades del plan esencial de aseguramiento universal. Rev Peru Med Exp Salud Publica. 2009;26(2):222-31.

2. Instituto Nacional de Salud Mental. Estudio epidemiológico metropolitano en salud mental 2002. Informe general. Anales de Salud Mental. 2002;18(12):1-199.

3. Instituto Nacional de Salud Mental. Estudio epidemiológico de salud mental en la sierra peruana 2003. Informe general. Anales de Salud Mental. 2003;19(1-2):1-216.

4. Instituto Nacional de Salud Mental. Estudio epidemiológico de salud mental en la selva peruana 2004. Informe general. Anales de Salud Mental. 2004;21(1-2):1-213.

5. Kessler RC, Angermeyer M, Anthony JC, DE Graaf R, Demyttenaere K, Gasquet I, et al. Lifetime prevalence and age-of-onset distributions of mental disorders in the World Health Organization's World Mental Health Survey Initiative. World Psychiatry. 2007;6(3):168-76.

6. kessler RC, Ustun $\mathrm{TB}$, editors. The WHO World Mental Health Surveys: Global Perspectives on the Epidemiology of Mental Disorders. New York: Cambridge University Press; 2008.

7. Instituto Nacional de Estadística e Informática. Censos Nacionales 2007: 
XI de Población y VI de Vivienda. Lima: Instituto Nacional de Estadística e Informática; 2008.

8. Kessler RC, Ustun TB. The World Mental Health (WMH) Survey Initiative Version of the World Health Organization (WHO) Composite International Diagnostic Interview (CIDI). Int J Methods Psychiatr Res. 2004;13(2):93-121.

9. Medina-Mora ME, Borges G, Benjet C, Lara C, Berglund P. Psychiatric disorders in Mexico: lifetime prevalence in a nationally representative sample. $\mathrm{Br}$ J Psychiatry. 2007;190:521-8.

10. Demyttenaere K, Bruffaerts R, PosadaVilla J, Gasquet I, Kovess V, Lepine JP, et al. Prevalence, severity, and unmet need for treatment of mental disorders in the World Health Organization World Mental Health Surveys. JAMA. 2004;291(21):2581-90.

11. Fiestas F, Radovanovic M, Martins SS, Medina-Mora ME, Posada-Villa J, Anthony JC. Cross-national differences in clinically significant cannabis problems: epidemiologic evidence from 'cannabis-only' smokers in the United States, Mexico, and Colombia. BMC Public Health. 2010;10:152. doi: 10.1186/1471-2458-10-152.

12. American Psychiatric Association. Diagnostic and Statistic Manual of Mental Disorders. 4th ed. Washington, DC: American Psychiatric Association; 1994.

13. Narrow WE, Rae DS, Robins LN, Regier DA. Revised prevalence estimates of mental disorders in the United States: using a clinical significance criterion to reconcile 2 surveys' estimates. Arch Gen Psychiatry. 2002;59(2):115-23.

14. Degenhardt L, Bohnert KM, Anthony JC. Case ascertainment of alcohol dependence in general population surveys: 'gated' versus 'ungated' approaches. Int J Methods Psychiatr Res. 2007;16(3):111-23.

15. Degenhardt L, Bohnert KM, Anthony JC. Assessment of cocaine and other drug dependence in the general population: "gated" versus "ungated" approaches. Drug Alcohol Depend. 2008;93(3):227-32.

16. Degenhardt L, Cheng H, Anthony JC. Assessing cannabis dependence in community surveys: methodological issues. Int J Methods Psychiatr Res. 2007;16(2):43-51.

17. Efron B. Logistic regression, survival analysis, and the Kaplan-Meier curve. J Am Statist Assoc. 1988;83:414-25.

18. Berglund PA. Analysis of complex sample survey data using the SURVEYMEANS and SURVEYREG procedures and macro coding. Ann Arbor: University of Michigan; 2002.

19. Wittchen HU. Reliability and validity studies of the WHO--Composite International Diagnostic Interview (CIDI): a critical review. J Psychiatr Res. 1994;28(1):57-84.

20. Kessler RC, Abelson J, Demler O, Escobar JI, Gibbon M, Guyer ME, et al. Clinical calibration of DSM-IV diagnoses in the World Mental Health (WMH) version of the World Health
Organization (WHO) Composite International Diagnostic Interview (WMHCIDI). Int J Methods Psychiatr Res. 2004;13(2):122-39.

21. Colombia, Ministerio de la Proyección Social. Estudio Nacional de Salud Mental-Colombia 2003. Cali: Ministerio de la Proyección Social; 2005.

22. Pennell BE, Bowers A, Carr D, Chardoul S, Cheung GQ, Dinkelmann $\mathrm{K}$, et al. The development and implementation of the National Comorbidity Survey Replication, the National Survey of American Life, and the National Latino and Asian American Survey. Int J Methods Psychiatr Res. 2004;13(4):241-69.

23. Bromet E, Andrade LH, Hwang I, Sampson NA, Alonso J, de Girolamo $\mathrm{G}$, et al. Cross-national epidemiology of DSM-IV major depressive episode. BMC Med. 2011;9:90. doi: 10.1186/1741-7015-9-90.

24. Fiestas F, Ladd G, Gallo C, Guimas B, Poletti G, Vaisberg A. La patología afectiva, ansiosa y somática en la América Andina. En: Ruiz P, Casas M, editors. Salud Mental en el paciente de América Andina. Barcelona: Editorial Glosa; 2009.

Correspondencia: Fabián Fiestas

Dirección: Cápac Yupanqui 1400 - Jesús Maria, Lima 11, Perú

Teléfono: (+511) 997-523318

Correo electrónico:fabianfiestas@yahoo.com

\section{Consulte la versión electrónica de la Revista Peruana de Medicina Experimental y Salud Pública en}

\section{wWw.scopus.com}

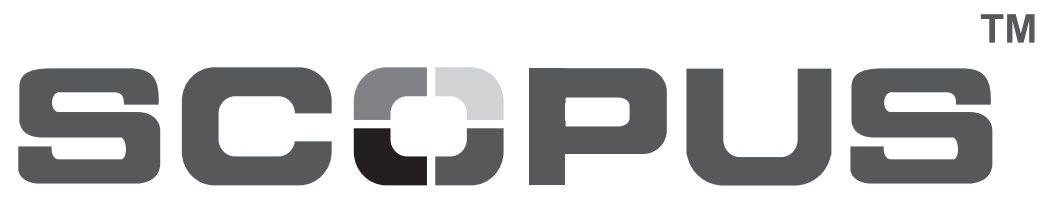

